\title{
Propofol post-conditioning protects the blood brain barrier by decreasing matrix metalloproteinase- 9 and aquaporin- 4 expression and improves the neurobehavioral outcome in a rat model of focal cerebral ischemia-reperfusion injury
}

\author{
FENG-TAO JI ${ }^{*}$, JIAN-JUN LIANG ${ }^{*}$, LI-PING MIAO, QIANG WU and MING-HUI CAO \\ Department of Anesthesiology, Sun Yat-Sen Memorial Hospital of Sun Yat-Sen University, \\ Guangzhou, Guangdong 510120, P.R. China
}

Received February 15, 2014; Accepted November 3, 2014

DOI: $10.3892 / \mathrm{mmr} .2015 .3585$

\begin{abstract}
Propofol, an intravenous anesthetic, inhibits neuronal apoptosis induced by ischemic stroke, protects the brain from ischemia/reperfusion injury and improves neuronal function. However, whether propofol is able to protect the blood brain barrier (BBB) and the underlying mechanisms have remained to be elucidated. In the present study, a rat model of cerebral ischemia/reperfusion was established, using a thread embolism to achieve middle cerebral artery occlusion. Rats were treated with propofol (propofol post-conditioning) or physiological saline (control) administered by intravenous injection $30 \mathrm{~min}$ following reperfusion. Twenty-four hours following reperfusion, neurobehavioral manifestations were assessed. The levels of cephaloedema, damage to the BBB and expression levels of matrix metalloproteinase-9 (MMP-9), aquaporin-4 (AQP-4) and phosphorylated c-Jun N-terminal kinase ( $\mathrm{pJNK}$ ) were determined in order to evaluate the effects of propofol on the BBB. In comparison to the cerebral ischemia/reperfusion group, the levels of brain water content and Evans blue content, as well as the expression levels of MMP-9, AQP-4 and pJNK were significantly reduced in the propofol post-conditioning group. These results indicated that propofol post-conditioning improved the neurobehavioral manifestations and attenuated the BBB damage and cephaloedema induced following cerebral ischemia/reperfusion. This effect may be due to the inhibition of MMP-9 and AQP-4 expression, and the concurrent decrease in JNK phosphorylation.
\end{abstract}

Correspondence to: Dr Feng-Tao Ji, Department of Anesthesiology, Sun Yat-Sen Memorial Hospital of Sun Yat-Sen University, 107 Yan Jiang West Road, Guangzhou, Guangdong 510120, P.R. China

E-mail: jifengtao@yeah.net

${ }^{*}$ Contributed equally

Key words: propofol, cerebral ischemia, ischemia reperfusion injury, blood brain barrier, matrix metalloproteinase, aquaporin-4, c-Jun N-terminal kinase

\section{Introduction}

Reperfusion of ischemic brain tissues is an effective protocol for the treatment of stroke. However, the aggravation of ischemia and hypoxia as well as the necrosis/apoptosis of neurons in the peripheral zone destroys the normal structure and function of brain tissues (1). The underlying mechanism of this effect involves the release of oxygen free radicals and excitatory amino acids as well as the activation of apoptosis-associated genes (including an increase in the expression of pro-apoptotic and a decrease in anti-apoptotic genes) (1). The destruction of the blood brain barrier (BBB) further induces cerebral edema and normal brain-tissue damage. These effects have markedly influenced the efficacy of therapies for cerebral ischemia/reperfusion injury (2). Numerous studies have shown that during cerebral ischemia in the $\mathrm{BBB}$, matrix metalloproteinase-9 (MMP-9) and aquaporin-4 (AQP-4) have crucial roles $(3,4)$ in the development of cerebral edema. MMP-9 is able to degrade the extracellular matrix and BBB, influence the permeability of blood vessels and participate in the induction of vasogenic brain edema (3). AQP-4 regulates water metabolism, water distribution and osmotic pressure (4). Furthermore, the c-Jun $\mathrm{N}$-terminal kinase (JNK) pathway is involved in the activation of MMP-9 and AQP-4 $(5,6)$.

Propofol, which is clinically used as an anesthetic, reduces cerebral blood flow, oxygen consumption and intracranial pressure (7). A previous study has indicated that propofol pre- and post-conditioning treatments may protect tissues from cerebral ischemia-reperfusion injury. Propofol inhibits the activation of proapoptotic genes, thereby reducing neuronal apoptosis and improving prognosis (7-9). However, previous studies have focused on the protective effects of propofol on neurons and the mechanisms underlying these effects, whereas there are few studies regarding the effects of propofol on the BBB and its underlying mechanisms. To the best of our knowledge, no study has been performed investigating the effects of propofol on the expression of MMP-9, AQP-4 and phosphorylated JNK following cerebral ischemia/reperfusion.

In the present study, an established rat model of cerebral ischemia/reperfusion was treated with propofol administered via intravenous injection. The condition of the BBB, level of 
cephaloedema and neural functions of propofol-treated rats were assessed and scored to evaluate the protective effects of propofol post-conditioning. The expression levels of MMP-9 and AQP-4 and the phosphorylation level of pJNK were also determined in order to elucidate the mechanism underlying the protective effects of propofol on the BBB.

\section{Materials and methods}

Drugs and reagents. The drugs and reagents used in the present study included propofol (Diprivan; AstraZeneca, London, UK), Evans blue (EB) and dimethylformamide (Sigma-Aldrich, St. Louis, MO, USA). Antibodies included rabbit anti-mouse MMP-9 polyclonal antibody and JNK/pJNK polyclonal antibody (Santa Cruz Biotechnology, Inc., Dallas, TX, USA) rabbit anti-mouse AQP-4 antibody (Chemicon ${ }^{\circledR}$, EMD Millipore, Billerica, MA, USA) and rabbit anti-mouse $\beta$-actin antibody (Sigma-Aldrich). A western blotting kit was purchased from KPL (Gaithersburg, MD, USA).

Experimental animal selection and grouping. Ninety-six healthy adult Wistar rats, weighing 260-300 g and aged 4-6 weeks (Experimental Animal Centre of Zhongshan University, Guangzhou, China) were kept in a $12 \mathrm{~h} / 12 \mathrm{~h}$ light/dark cycle at $28-32^{\circ} \mathrm{C}$ with free access to food and water. Rats were randomly divided into four groups: The sham operation group (group $\mathrm{S}, \mathrm{n}=24$ ), the ischemia reperfusion group (group I, n=24) and two propofol-treated groups, which included group P1 (20 mg/kg/h profopol, $\mathrm{n}=24)$ and group P2 (40 mg/kg/h profopol, $\mathrm{n}=24$ ). The study was approved by the Animal Care Committee of Sun Yat-sen University (Guangzhou, China) and performed in strict accordance with the National Institutes of Health Guide for the Use of Laboratory Animals.

Preparation of cerebral ischemia/reperfusion rat model and assessment of neural function. The rats were subjected to fasting without drinking for $12 \mathrm{~h}$ prior to surgery. Anesthesia was induced in a Plexiglass chamber with $4 \%$ halothane (Sigma-Aldrich), followed by tracheal intubation and mechanical ventilation with $1.3 \%$ halothane in $30 \% \mathrm{O}_{2} / 70 \% \mathrm{~N}_{2} \mathrm{O}$. The left femoral artery was cannulated to monitor blood pressure and spontaneous breath was conserved. The rat was prepared for surgery by the removal of hair in the middle of the neck in the supine position. Subsequently the skin was prepared with $75 \%$ alcohol and a surgical incision was made at the flank of the neck. The right common carotid artery, right external carotid artery, right internal carotid artery and wing palatal artery were isolated. The external carotid and wing palatal arteries were ligatured and the right common carotid artery was blocked by a bulldog clamp, while the right external carotid artery was punctured $0.5 \mathrm{~cm}$ from the ligation at the proximal end to heart. A nylon thread was inserted into the wound and pushed through the right internal carotid artery in the direction of the brain and the opposite side of the artery was pierced 18-20 mm further down. The middle cerebral artery was then blocked. Following surgery, cerebral blood flow was required to be reduced to $\sim 30 \%$; otherwise, the rat model was considered unsuccessful and removed (10). Two hours following the arterial block, the thread was removed, the residual end of the artery was fastened and the skin and subcutaneous tissue were sutured. Once the rats regained consciousness, behavioral disorders were assessed according to the Longa method (11) using the following scale: No visible neural function loss, 0 ; left forepaw unable to straighten, 1; walking impaired with an inclination towards ischemic hemisphere, 2; repeated left turning, 3; falling left, 4; unable to walk, 5 .

Drug treatment. The $\mathrm{P} 1$ and $\mathrm{P} 2$ groups were treated with $20 \mathrm{mg} / \mathrm{kg} / \mathrm{h}$ or $40 \mathrm{mg} / \mathrm{kg} / \mathrm{h}$ propofol, respectively, for $30 \mathrm{~min}$ via the tail vein, 30 min following reperfusion. Group I was treated with an equal amount of physiological saline (SigmaAldrich)and in group S, the incision was made but no arterial occlusion was performed.

The water content of the ischemic hemisphere of the brain was detected using the wet/dry weight ratio method with the following formula: Water content $(\%)=100 x$ [(wet weight)-(dry weight)]/wet weight.

Detection of $E B$. The permeability of the BBB was determined according to the protocol described by Uyama et al (12), by the detection of EB fluorescence. Twenty-three hours following reperfusion, rats were anesthetized with $10 \%$ chloral hydrate and $2 \% \mathrm{~EB}(3 \mathrm{ml} / \mathrm{kg})$ was injected into the femoral vein. One hour later, the ventriculus sinister was washed with physiological saline for $0.5 \mathrm{~h}$. The brain was removed by decollation and surface moisture was blotted with filter paper. The cerebral cortex of the ischemic side was excised and the wet weight was measured with an electronic balance prior to incubation with $4 \mathrm{ml}$ dimethylformamide (Sigma-Aldrich) at $50^{\circ} \mathrm{C}$ for $48 \mathrm{~h}$ followed by centrifugation at $1509 \mathrm{x}$ g for $15 \mathrm{~min}$. The absorbency of the supernatant at $620 \mathrm{~nm}$ was determined using a Hitachi U2001 (Hitachi, Ltd, Tokyo, Japan). The content of EB $(\mu \mathrm{g} / \mathrm{g})$ was subsequently calculated according to the standard curve method (12).

Immunohistochemical examination. Half of the animals in each group were randomly selected to be sacrificed by administration of 5\% halothane (Sigma-Aldrich) and fixed in the dorsal position immediately following behavioral testing. The rat hearts were exposed by opening the chest from the xiphisternum and were perfused through the ascending aorta with $150 \mathrm{ml}$ saline followed by $400 \mathrm{ml} \mathrm{4 \%}$ paraformaldehyde. Following perfusion, the brain was removed, fixed in $4 \%$ paraformaldehyde (Sigma-Aldrich) overnight and subsequently transferred to $30 \%$ sucrose (Sigma-Aldrich) and incubated for 3-5 days. The damaged brain area was dissected into $1-\mathrm{mm}$ coronal sections. Sequential $10-\mu \mathrm{m}$ coronal sections were cut from the sections (from bregma -2.3 to $-2.45 \mathrm{~mm}$ ) by cryomicrotomy (CM1900; Leica Microsystems GmbH, Wetzlar, Germany), the sections were treated with $0.3 \% \mathrm{H}_{2} \mathrm{O}_{2}$ (Sigma-Aldrich) in methanol for $30 \mathrm{~min}$, hydrated gradually in distilled water and incubated for $2 \mathrm{~h}$ with $5 \%$ goat serum (Sigma-Aldrich) to block nonspecific immune reactions. The sections were subsequently incubated at $4^{\circ} \mathrm{C}$ overnight with rabbit polyclonal anti-MMP-9 or anti-AQP-4 antibodies (1:2,000). Following washing with Tris-buffered saline (Sigma-Aldrich), the sections were incubated with biotinylated goat anti-rabbit immunoglobulin G ( $\operatorname{IgG})$ (1:200; Zhongshan Golden Bridge Biotechnology Co., Ltd, Beijing, China) for $2 \mathrm{~h}$ 
Table I. Effect of propofol post-conditioning on neurobehavioral scores, water content and EB content $24 \mathrm{~h}$ following reperfusion.

\begin{tabular}{lccc}
\hline Group & Neurobehavioral scores $[M(Q), \mathrm{n}=18]$ & Water content $(\%, \mathrm{n}=6)$ & EB content $(\mu \mathrm{g} / \mathrm{g}, \mathrm{n}=6)$ \\
\hline S & 0 & $77.41 \pm 0.19$ & $0.54 \pm 0.31$ \\
I & $3.0(1.0)^{\mathrm{a}}$ & $86.51 \pm 0.53^{\mathrm{a}}$ & $12.65 \pm 3.14^{\mathrm{a}}$ \\
P1 & $2.0(1.0)^{\mathrm{a}, \mathrm{b}}$ & $82.72 \pm 0.86^{\mathrm{a}, \mathrm{b}}$ & $5.72 \pm 2.78^{\mathrm{a}, \mathrm{b}}$ \\
P2 & $2.0(0.5)^{\mathrm{a}, \mathrm{b}}$ & $81.25 \pm 0.67^{\mathrm{a}, \mathrm{b}}$ & $4.76 \pm 2.17^{\mathrm{a}, \mathrm{b}}$ \\
\hline
\end{tabular}

${ }^{\mathrm{a}} \mathrm{P}<0.05$ vs. group $\mathrm{S} ;{ }^{\mathrm{b}} \mathrm{P}<0.05$ vs. group I. Values are expressed as the mean \pm standard deviation. EB, Evans blue; $M(Q)$, median (quartile); $\mathrm{S}$, sham group; I, ischemia/reperfusion group; P1, propofol-treated group (20 mg/kg); P2, propofol-treated group (40 mg/kg).

followed by incubation with avidin-biotinperoxidase complex (1:200; Zhongshan Golden Bridge Biotechnology Co.) for $2 \mathrm{~h}$. Finally, the sections were exposed to $0.01 \%$ 3,3'-diaminobenzidine (Sigma-Aldrich) for 0.5-2 min, followed by examination under a light microscope (Olympus BX51; Olympus Corp., Tokyo, Japan). The optical density of MMP-9 and AQP-4 immunoreactivity was evaluated in an area of $1 \mathrm{x} 1 \mathrm{~mm}^{2}$ in the boundary zone.

Western blot analysis. Twenty-four hours following reperfusion, rats were sacrificed. The brains were removed and immediately cut into two 2-mm sections on dry ice, excluding $4 \mathrm{~mm}$ of the rostral tissue. Slices were cut into the right and left hemispheres, and the right striatum and parietal cortex were isolated and homogenized in tissue lysate buffer $(10 \mathrm{ml} / \mathrm{mg}$ tissue) containing a mixture of proteinase inhibitors (Thermo Fisher Scientific China Co., Ltd, Beijing, China), prior to dispersion by sonication. The homogenate was centrifuged at $2,250 \mathrm{x}$ g for $30 \mathrm{~min}$ at $4^{\circ} \mathrm{C}$, and the supernatant was saved. A bicinchoninic acid assay (Pierce Biotechnology, Inc.; Thermos Fisher Scientific, Rockford, IL, USA) was used to determine the protein concentration. Proteins $(30 \mu \mathrm{g} /$ well) were separated on using $10 \%$ SDS-PAGE and transferred to nitrocellulose membranes (Sigma-Aldrich) at $100 \mathrm{mV}$. The membranes were blocked with 5\% non-fat milk (Sigma-Aldrich) in phosphate-buffered saline with $0.1 \%$ Tween-20 (Sigma-Aldrich) for $1 \mathrm{~h}$ at room temperature and incubated overnight at $4^{\circ} \mathrm{C}$ with rabbit anti-mouse MMP-9 polyclonal antibody (1:2,000; Santa Cruz Biotechnology, Inc.), rabbit anti-mouse AQP-4 antibody (1:2,000; Abcam, Cambridge, MA, USA) or rabbit polyclonal anti-pJNK antibody (1:2,000; Santa Crux Biotechnology, Inc.). Following three washes in Tris-buffered saline for $5 \mathrm{~min}$, the blots were incubated for $1 \mathrm{~h}$ at room temperature with the appropriate horseradish peroxidase-conjugated secondary antibody (1:3,000; Santa Cruz Biotechnology, Inc.). MMP-9, AQP-4 and pJNK were subsequently visualized by incubation with enhanced chemiluminescence reagent (Thermo Fisher Scientific, Waltham, MA, USA) for $1 \mathrm{~min}$, and exposure onto hyperfilm (Sigma-Aldrich) for 1-10 min. Membranes which were positive for JNK were re-blocked with $5 \%$ non-fat milk for $1 \mathrm{~h}$ at room temperature and incubated with rabbit polyclonal anti-JNK antibody (Santa Cruz Biotechnology, Inc.) overnight at $4^{\circ} \mathrm{C}$. Visualization of JNK was performed as described above. Image J 1.35 software (National Institutes of Health, Bethesda, MD, USA) was used to analyze the relative density values.
Statistical analysis. Data were analyzed using SPSS 16.0 (SPSS, Inc., Chicago, IL, USA). Values are expressed as the mean \pm standard deviation. Differences between groups were analyzed using one-way analysis of variance and multiple comparisons were made using the least significant difference test. Data of skewed distribution are presented as the median. $\mathrm{P}<0.05$ was considered to indicate a statistically significant difference between values.

\section{Results}

Propofol post-conditioning improves neurobehavioral scores and decreases water-and EB content $24 \mathrm{~h}$ following reperfusion. The assessment of neurological function indicated that all experimental animals exhibited post-ischemic neurological deficits. Rats in groups I, P1, and P2 scored significantly higher than those in group $\mathrm{S}(\mathrm{P}<0.05)$. Compared with the rats in group I, those in groups $\mathrm{P} 1$ and P2 scored significantly lower $(\mathrm{P}<0.05)$. The experimental groups demonstrated higher water content and EB content in the ischemic hemisphere in comparison to that of group $\mathrm{S}$ $(\mathrm{P}<0.05)$. The water and EB content were significantly reduced in groups $\mathrm{P} 1$ and $\mathrm{P} 2$ compared to those in group I $(\mathrm{P}<0.05$; Table I).

MMP-9 and AQP-4 expression in the ischemic hemisphere are attenuated following propofol treatment. Immunohistochemical analysis indicated that the expression levels of MMP-9 and AQP-4 in ischemic brain tissue in group I were significantly increased compared to those in group $\mathrm{S}$ $(\mathrm{P}<0.05)$. Following propofol post-conditioning, the expression levels of MMP-9 and AQP-4 were significantly decreased in groups $\mathrm{P} 1$ and $\mathrm{P} 2$ compared with those in group $\mathrm{I}(\mathrm{P}<0.05)$, although expression levels remained significantly higher than those in group $\mathrm{S}(\mathrm{P}<0.05$; Figs. 1 and 2$)$.

Propofol post-conditioning decreases the expression of $M M P-9, A Q P-4$ and $p J N K$ in brain tissues. Twenty-four hours following ischemia/reperfusion, the expression levels of MMP-9, AQP-4 and the levels of pJNK were significantly increased. Following treatment with propofol, the expression levels of MMP-9, AQP-4 and pJNK in groups P1 and P2 were significantly decreased $(\mathrm{P}<0.05)$. Furthermore, the expression levels of MMP-9, AQP-4 and pJNK were significantly decreased in group P2 compared with those in group P1 $(\mathrm{P}<0.05$; Figs. 3-5). 

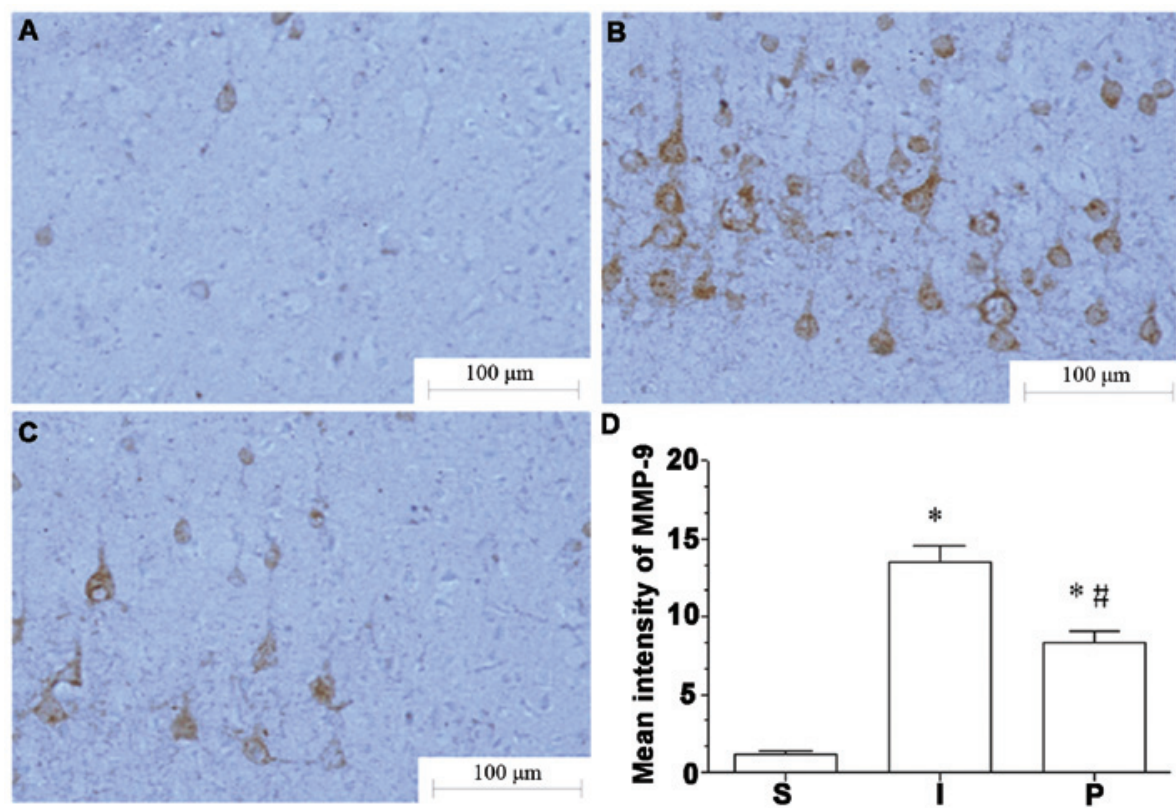

Figure 1. Effect of propofol post-conditioning on expression levels of MMP-9 $24 \mathrm{~h}$ following reperfusion. Staining of MMP-9-positive cells in brain tissue of (A) group S, (B) group I and (C) group P. (D) Quantitative analysis of MMP-9-positive cells. Values are expressed as the mean \pm standard deviation, $\mathrm{n}=6$. ${ }^{*} \mathrm{P}<0.05$ vs, group S, " $\mathrm{P}<0.05$ vs. group I. MMP-9, matrix metalloproteinase-9; S, sham group; I, ischemia/reperfusion group; P, propofol-treated groups P1 and P2.
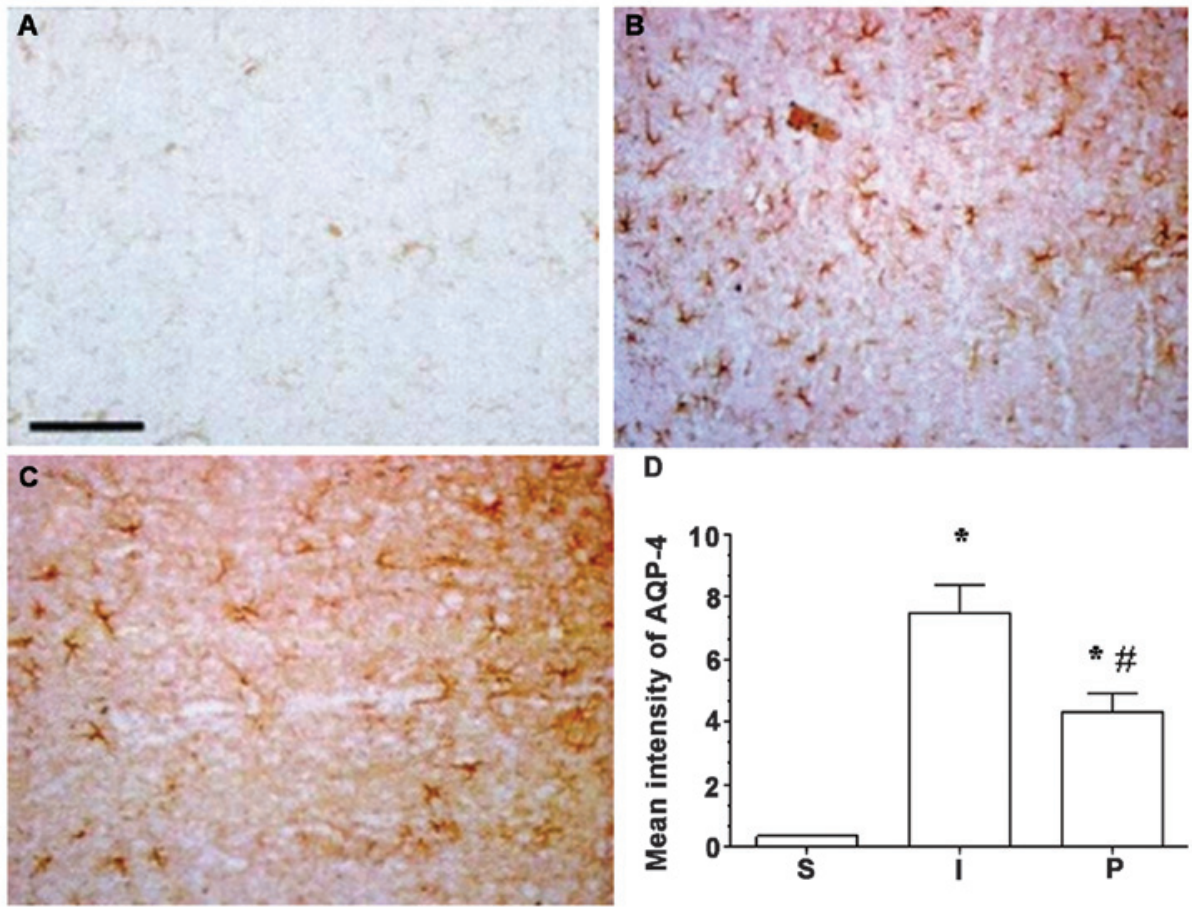

Figure 2. Effect of propofol post-conditioning on expression levels of AQP-4 $24 \mathrm{~h}$ following reperfusion. Staining figure of AQP-4-positive cells in brain tissue of (A) group S, (B) group I and (C) group P. (D) Quantitative analysis of AQP-4-positive cells. Values are expressed as the mean \pm standard deviation, $\mathrm{n}=6$. " $\mathrm{P}<0.05$ vs. group S, "P<0.05 vs. group I. Scale bar, $50 \mu \mathrm{m}$. AQP-4, aquaporin-4; S, sham group; I, ischemia/reperfusion group; P, propofol-treated groups P1 and P2.

\section{Discussion}

The middle cerebral artery is prone to ischemic cerebrovascular disease. In a previous study, a model of cerebral ischemia/reperfusion was established using the Longa method (11), which was selected for the high success rate, reproducibility and low risk of complications. This established model effectively simulated the clinical and pathological characteristics of cerebral infarction. In the present study, rats with cerebral ischemia/reperfusion scored significantly lower in the assessment of neural function. Cerebral infarction was observed in the ischemic hemisphere, which indicated that the model reflected the clinical and pathological characteristics of cerebral infarction. Vascular occlusion resulted in cerebral circulatory dysfunction, and therefore cerebral ischemia and hypoxia irreversibly damaged neurons in the affected 

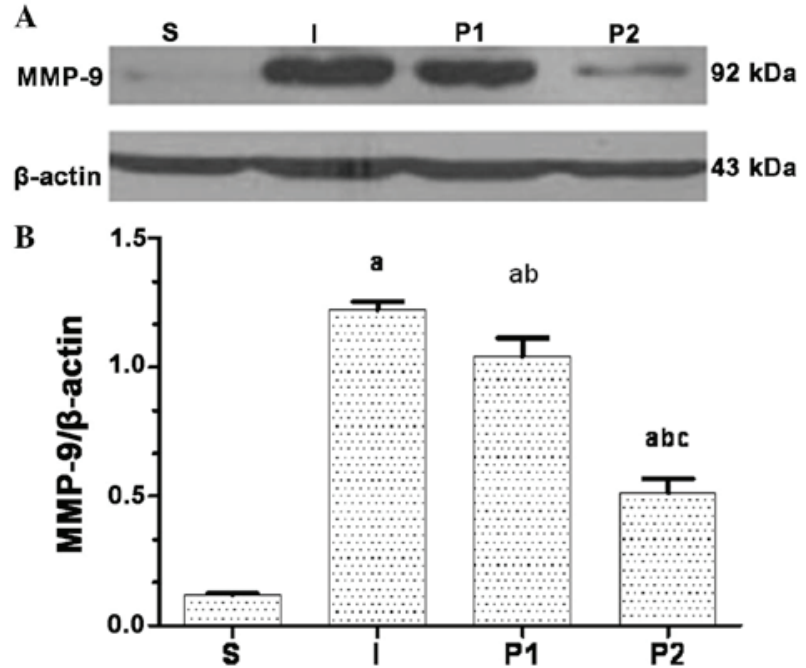

Figure 3. Effect of propofol post-conditioning on the expression of MMP-9 $24 \mathrm{~h}$ following reperfusion. (A) Western blot analysis of MMP-9 and $\beta$-actin in the different groups. (B) Graph showing the ratio of MMP-9/ $\beta$-actin. Values are expressed as the mean \pm standard deviation, $n=6$. ${ }^{a} \mathrm{P}<0.05$ vs. group $\mathrm{S}$, ${ }^{\mathrm{b}} \mathrm{P}<0.05$ vs. group I, ${ }^{\mathrm{C}} \mathrm{P}<0.05$ vs. group $\mathrm{P} 1$. MMP-9, matrix metalloproteinase-9; $\mathrm{S}$, sham group; I, ischemia/reperfusion group; P1, propofol-treated group (20 mg/kg); P2, propofol-treated group (40 mg/kg).
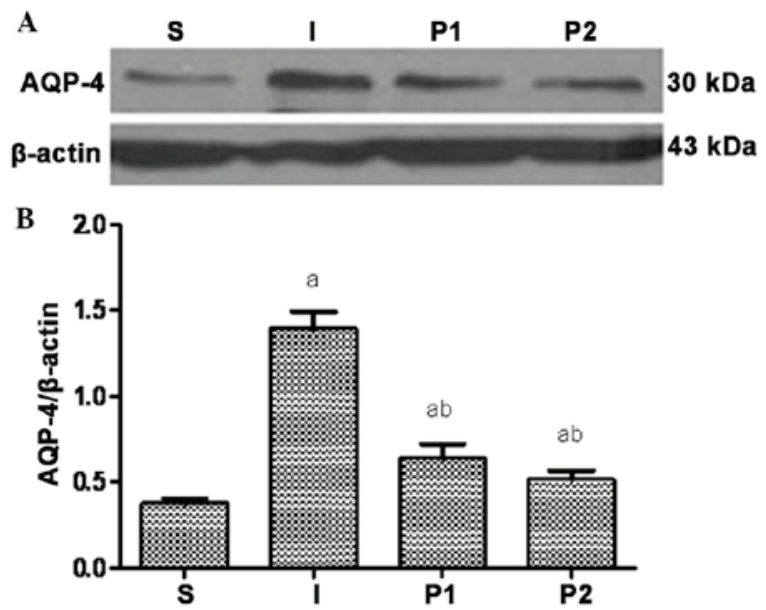

Figure 4. Effect of propofol post-conditioning on the expression of AQP-4 $24 \mathrm{~h}$ following reperfusion. (A) Western blot analysis of AQP-4 and $\beta$-actin in the different groups. (B) Graph showing the ratio of AQP- $4 / \beta$-actin. Values are expressed as the mean \pm standard deviation, $n=6$. ${ }^{a} \mathrm{P}<0.05$ vs. group $\mathrm{S}$, ${ }^{b} \mathrm{P}<0.05$ vs. group I. AQP-4, aquaporin-4; $\mathrm{S}$, sham group; I, ischemia/reperfusion group; P1, propofol-treated group ( $20 \mathrm{mg} / \mathrm{kg})$; P2, propofol-treated group $(40 \mathrm{mg} / \mathrm{kg})$.

part of the brain. Furthermore, damage to the BBB induced successive cerebral edema, which further impaired brain tissues. A significant improvement in neural function was observed following propofol post-conditioning in cerebral ischemia/reperfusion rats and the water content of the brain was also decreased. These results demonstrated that propofol treatment may protect against cerebral ischemia/reperfusion damage, which was in agreement with the results reported in a previous paper (9).

The aquaporins are a family of water-selective cell membrane transporters. AQP-4 widely exists in the central
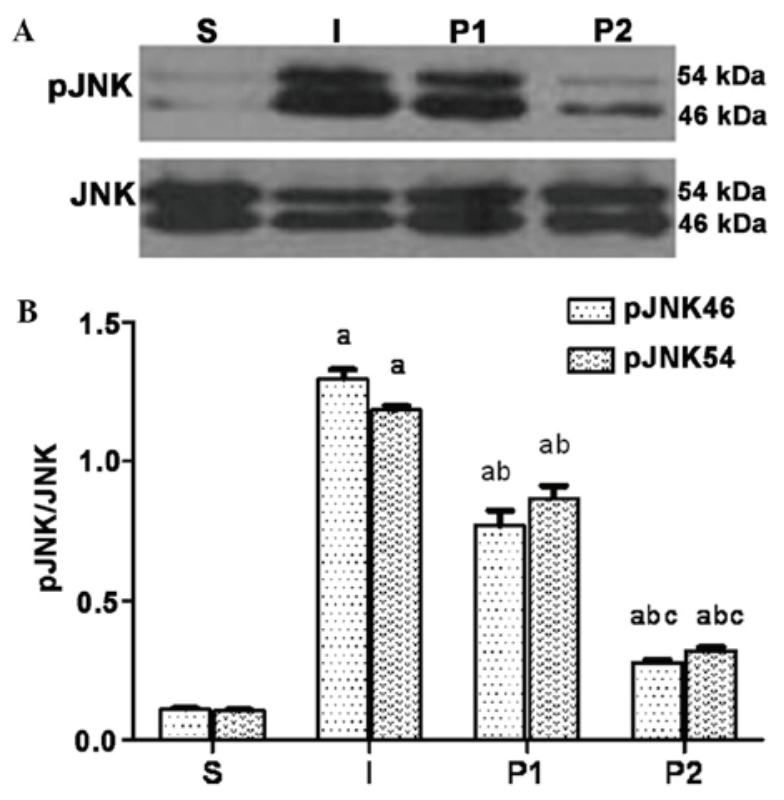

Figure 5. Effect of propofol post-conditioning on phosphorylation of JNK $24 \mathrm{~h}$ following reperfusion. (A) Western blot analysis of pJNK and JNK in the different groups. (B) Graph showing the ratio of $\mathrm{pJNK} / \mathrm{JNK}$. Values are expressed as the mean \pm standard deviation, $\mathrm{n}=6 .{ }^{\mathrm{a}} \mathrm{P}<0.05$ vs. group $\mathrm{S},{ }^{\mathrm{b}} \mathrm{P}<0.05$ vs. group I, ${ }^{c} \mathrm{P}<0.05$ vs. group $\mathrm{P} 1$. pJNK, phosphorylated c-Jun N-terminal kinase; S, sham group; I, ischemia/reperfusion group; P1, propofol-treated group (20 mg/kg); P2, propofol-treated group ( $40 \mathrm{mg} / \mathrm{kg})$.

nervous system, and localizes to the membrane of astrocytes, subarachnoid space, glial cells surrounding vessels, ependymal cells and the choroid plexus (13). AQP-4 is a critical component of integrated water and potassium homeostasis (14), and has important roles in water metabolism, localization and osmotic pressure (4). AQP-4 knock-out rats were found to have improved cephaloedema following cerebral ischemic stroke (15). MMP-9 is a type of matrix metalloproteinase that is able to degrade the basal lamina and disrupt the BBB. MMP-9 is involved in the pathophysiological process of cerebral ischemia via the degradation of tight junctions between the basal lamina and endothelial cells, which results in the destruction of the BBB (16). MMP-9 has a pivotal role in BBB proteolysis (17), and increased MMP-9 expression and activation increases the permeability of the BBB and may therefore exacerbate cephaloedema $(3,18,19)$. Furthermore, during cerebral ischemia hypoxia-inducible factor-1 $\alpha$ may damage the BBB by activating the expression of AQP-4 and MMP-9 and aggravating cerebral edema. Inhibitors of AQP-4 and MMP-9 may protect the BBB to a certain extent (20). The extent of brain edema observed in cerebral ischemia/reperfusion animals was significantly reduced following inhibition, and the animals demonstrated an improved prognosis (21-23). These results indicated that the mechanism underlying BBB protection may be associated with the inhibition of AQP-4 and MMP-9 function. Studies have additionally demonstrated that propofol inhibited the expression of AQP-4 in astroglia in vitro (23) and decreased the expression of MMP-9 in vivo (24). In the present study, the expression levels of AQP-4 and MMP-9 were markedly lower in groups P1 and P2 than those in group S, indicating that treatment with propofol inhibited the expression of these proteins. 
The activity of AQP-4 and MMP-9 is influenced by numerous factors. Studies have indicated that damage to the central nervous system may activate the expression of MMP-9 and AQP-4 via the JNK pathway $(5,6)$. JNK is a member of the mitogen-activated protein kinase (MAPK) family, which are involved in mediating the inflammatory response following cerebral ischemia, cell factors, cell proliferation and differentiation as well as the process of oxidant stress $(5,6)$. The activation of JNK induces the phosphorylation of downstream factors, including c-Jun, protein 53 and activator protein 1, as well as modulating gene expression, protein synthesis and neuronal apoptosis (25-28). Animal experiments have revealed that the phosphorylation of JNK is involved in neuronal apoptosis, and that the inhibition of JNK phosphorylation is beneficial for reducing the infarct area and improving prognosis following cerebral ischemia (29). In vitro experiments demonstrated that propofol inhibited the activation of JNK and reduced the levels of cell death induced by oxidative stress (30). Previous studies demonstrated that intraperitoneal administration of $150 \mathrm{mg} / \mathrm{kg}$ propofol or intravenous administration of $20 \mathrm{mg} / \mathrm{kg}$ propofol significantly alleviated cerebral edema, decreased the infarction size during ischemia/reperfusion injury and improved the National Institutes of Health Stroke Scale (NIHSS) score in a rat model (9). Therefore, the dosage of propofol selected for use in the present study was reasonable and effective in accordance with previous experiments. The results of the present study indicated that the levels of pJNK in groups P1 and P2 were markedly lower than those in the ischemia/reperfusion group. Due to their similar chemical structures, propofol has analogous biochemical functions to those of Vitamin E, including anti-oxidative, mitochondrial protective and free-radical scavenging activity (31-33). Furthermore, propofol inhibits the phosphorylation of JNK $(30,34)$. These results therefore indicated that the mechanism underlying the inhibitory effect of propofol on AQP-4 and MMP-9 may be via inactivation of the JNK pathway.

The function of the BBB is to maintain the central nervous system and following ischemia/reperfusion, the structure and function of the BBB is altered. When the BBB is damaged, its permeability is increased, which allows factors that cannot cross the barrier under physiological conditions to cross the BBB (2). This process may induce or aggravate cephaloedema. The increased permeability of the BBB will additionally further increase leukocyte infiltration in the ischemic brain tissue, enhancing inflammation (35). EB is a classical indicator of BBB permeability (12). The complex of $\mathrm{EB}$ and plasma albumin (ESA) is unable to cross the $\mathrm{BBB}$ under normal conditions; however, when the BBB is damaged, ESA is able to invade and stain the brain. The infiltration of $\mathrm{EB}$ across the $\mathrm{BBB}$ is positively correlated with the extent of BBB destruction (12). Propofol decreased the expression of AQP-4 and MMP-9 in ischemia/reperfusion rats and downregulated the phosphorylation of JNK, therefore protecting the integrity of the BBB. The lower water content of groups P1 and P2 compared with that of group I indicated that propofol treatment may protect the brain by reducing the damage to the $\mathrm{BBB}$ and the extent of cephaloedema.

Propofol reduced the levels of cephaloedema and the destruction of the BBB following ischemia/reperfusion and improved the score on the NIHSS. The underlying mechanism involved the inhibition of AQP-4, MMP-9 and phosphorylation of JNK. In conclusion, the results of the present study indicated that propofol exerts a neuroprotective effect via protection of the BBB.

\section{Acknowledgements}

The present study was supported by the Guangdong Province Science and Technology Plan Project, Guangzhou, China (no. 2010B080701004) and the Guangdong Province Medical Research Fund (no. B2010092).

\section{References}

1. Ginsberg MD: Role of free radical reactions in ischaemic brain injury. Drug News Perspect 14: 81-88, 2001.

2. Pluta R: Pathological opening of the blood-brain barrier to horseradish peroxidase and amyloid precursor protein following ischemia-reperfusion brain injury. Chemotherapy 51: 223-226, 2005.

3. Tu XK, Yang WZ, Shi SS, et al: 5-lipoxygenase inhibitor zileuton attenuates ischaemic brain damage: involvement of matrix metalloproteinase 9. Neurol Res 31: 848-852, 2009.

4. Papadopoulos MC and Verkman AS: Aquaporin-4 and brain edema. Pediatr Nephrol 22: 778-784, 2007.

5. Vikman P,AnsarS,Henriksson M,etal: Cerebral ischemiainduces transcription of inflammatory and extracellular-matrix-related genes in rat cerebral arteries. Exp Brain Res 183: 499-510, 2007.

6. Yatsushige H, Ostrowski RP, Tsubokawa T, et al: Role of c-jun N-terminal kinase in early brain injury after subarachnoid hemorrhage. J Neurosci Res 85: 1436-1448, 2007.

7. Engelhard K, Werner C, Eberspächer E, et al: Influence of propofol on neuronal damage and apoptotic factors after incomplete cerebral ischemia and reperfusion in rats: a long-term observation. Anesthesiology 101: 912-917, 2004.

8. Wang H, Luo M, Li C and Wang G: Propofol post-conditioning induced long-term neuroprotection and reduced internalization of AMPAR GluR2 subunit in a rat model of focal cerebral ischemia/reperfusion. J Neurochem 119: 210-219, 2011.

9. Wang HY, Wang GL, Yu YH and Wang Y: The role of phosphoinositide-3-kinase/Akt pathway in propofol-induced postconditioning against focal cerebral ischemia-reperfusion injury in rats. Brain Res 1297: 177-184, 2009.

10. Xing B, Chen $\mathrm{H}$, Zhang M, et al: Ischaemic post-conditioning protects brain and reduces inflammation in a rat model of focal cerebral ischemia/reperfusion. J Neurochem 105: 1737-1745, 2008.

11. Longa EZ, Weinstein PR, Carlson S and Cummins R: Reversible middle cerebral artery occlusion without craniotomy in rats. Stroke 20: 84-91, 1989.

12. Uyama O, Okamura N, Yanase M, et al: Quantitative evaluation of vascular permeability in the gerbil brain after transient ischemia using Evans blue fluorescence. J Cereb Blood Flow Metab 8: 282-284, 1988.

13. Zador Z, Stiver S, Wang V and Manley G: Role of aquaporin-4 in cerebral edema and stroke. Handb Exp Pharmacol 190: 159-170, 2009.

14. Ho JD, Yeh R, Sandstrom A, et al: Crystal structure of human aquaporin 4 at $1.8 \mathrm{~A}$ and its mechanism of conductance. Proc Natl Acad Sci USA 106: 7437-7442, 2009.

15. Manley GT, Fujimura M, Ma T, et al: Aquaporin-4 deletion in mice reduces brain edema after acute water intoxication and ischaemic stroke. Nat Med 6: 159-163, 2000.

16. Heo JH, Lucero J, Abumiya T, Koziol JA, Copeland BR and del Zoppo GJ: Matrix metalloproteinases increase very early during experimental focal cerebral ischemia. J Cereb Blood Flow Metab 19: 624-633, 2009.

17. Barr TL, Latour LL, Lee KY, et al: Blood-brain barrier disruption in humans is independently associated with increased matrix metalloproteinase-9. Stroke 41: e123-e128, 2010.

18. Lee K, Lee JS, Jang HJ, et al: Chlorogenic acid ameliorates brain damage and edema by inhibiting matrix metalloproteinase- 2 and 9 in a rat model of focal cerebral ischemia. Eur J Pharmacol 689: 89-95, 2012. 
19. Hartz AM, Bauer B, Soldner EL, et al: Amyloid- $\beta$ contributes to blood-brain barrier leakage in transgenic human amyloid precursor protein mice and in humans with cerebral amyloid angiopathy. Stroke 43: 514-523, 2012.

20. Wang Z, Meng CJ, Shen XM, et al: Potential contribution of hypoxia-inducible factor- $1 \alpha$, aquaporin- 4 , and matrix metalloproteinase-9 to blood-brain barrier disruption and brain edema after experimental subarachnoid hemorrhage. J Mol Neurosci 48 273-280, 2012.

21. Igarashi H, Huber VJ, Tsujita M and Nakada T: Pretreatment with a novel aquaporin 4 inhibitor, TGN-020, significantly reduces ischaemic cerebral edema. Neurol Sci 32: 113-116, 2011.

22. Hu Q, Chen C, Khatibi NH, et al: Lentivirus-mediated transfer of MMP-9 shRNA provides neuroprotection following focal ischaemic brain injury in rats. Brain Res 1367: 347-359, 2011.

23. Wang Z, Leng Y, Tsai LK, et al:Valproic acid attenuates blood-brain barrier disruption in a rat model of transient focal cerebral ischemia: the roles of HDAC and MMP-9 inhibition. J Cereb Blood Flow Metab 31: 52-57, 2011.

24. Zhu SM, Xiong XX, Zheng YY and Pan CF: Propofol inhibits aquaporin 4 expression through a protein kinase $\mathrm{C}$-dependent pathway in an astrocyte model of cerebral ischemia/reoxygenation. Anesth Analg 109: 1493-1499, 2009.

25. Deegan CA, Murray D, Doran P, et al: Anesthetic technique and the cytokine and matrix metalloproteinase response to primary breast cancer surgery. Reg Anesth Pain Med 35: 490-495, 2010.

26. Chang L and Karin M: Mammalian MAP kinase signalling cascades. Nature 410: 37-40, 2001

27. Tripathi A and Sodhi A: Growth hormone-induced production of cytokines in murine peritoneal macrophages in vitro: role of JAK/STAT, PI3K, PKC and MAP kinases. Immuno Biology 214: 430-440, 2009.
28. Park HS, Huh SH, Kim MS, et al: Nitric oxide negatively regulates c-jun $\mathrm{N}$-terminal kinase/stress-activated protein kinase by means of S-nitrosylation. Proc Natl Acad Sci USA 97: 14382-14387, 2000.

29. Zeng XW, Li MW, Pan J, et al: Activation of c-jun N-terminal kinase $1 / 2$ regulated by nitric oxide is associated with neuronal survival in hippocampal neurons in a rat model of ischemia. Chin Med J (Engl) 124: 3367-3372, 2011.

30. Murata Y, Fujiwara N, Seo JH, et al: Delayed inhibition of c-jun N-terminal kinase worsens outcomes after focal cerebral ischemia. J Neurosci 32: 8112-8115, 2012.

31. Shu L, Li T, Han S, et al: Inhibition of neuron-specific CREB dephosphorylation is involved in propofol and ketamine-induced neuroprotection against cerebral ischaemic injuries of mice. Neurochem Res 37: 49-58. 2012.

32. De La Cruz JP, Villalobos MA, Sedeno G, et al: Effect of propofol on oxidative stress in an in vitro model of anoxia-reoxygenation in the rat brain. Brain Res 800: 136-144, 1998.

33. Shibata H, Katsuki H, Okawara M, et al: c-jun N-terminal kinase inhibition and alpha-tocopherol protect midbrain dopaminergic neurons from interferon-gamma/lipopolysaccharide-induced injury without affecting nitric oxide production. J Neurosci Res 83: 102-109, 2006.

34. Corcoran TB, Engel A, Sakamoto H, et al: The effects of propofol on neutrophil function, lipid peroxidation and inflammatory response during elective coronary artery bypass grafting in patients with impaired ventricular function. Br J Anaesth 97: 825-831, 2006.

35. Ouyang YB, Voloboueva LA, Xu LJ and Giffard RG: Selective dysfunction of hippocampal CA1 astrocytes contributes to delayed neuronal damage after transient forebrain ischemia. J Neurosci 27: 4253-4260, 2007. 